\title{
On the Stability of Quadratic Functional Equations
}

\author{
Jung Rye Lee, ${ }^{1}$ Jong Su An, ${ }^{2}$ and Choonkil Park ${ }^{3}$ \\ ${ }^{1}$ Department of Mathematics, Daejin University, Kyeonggi 487-711, South Korea \\ ${ }^{2}$ Department of Mathematics Education, Pusan National University, Pusan 609-735, South Korea \\ ${ }^{3}$ Department of Mathematics, Hanyang University, Seoul 133-791, South Korea
}

Correspondence should be addressed to Jung Rye Lee, jrlee@daejin.ac.kr

Received 5 October 2007; Revised 27 November 2007; Accepted 4 January 2008

Recommended by Paul Eloe

Let $X, Y$ be vector spaces and $k$ a fixed positive integer. It is shown that a mapping $f(k x+y)+$ $f(k x-y)=2 k^{2} f(x)+2 f(y)$ for all $x, y \in X$ if and only if the mapping $f: X \rightarrow Y$ satisfies $f(x+y)+f(x-y)=2 f(x)+2 f(y)$ for all $x, y \in X$. Furthermore, the Hyers-Ulam-Rassias stability of the above functional equation in Banach spaces is proven.

Copyright (C) 2008 Jung Rye Lee et al. This is an open access article distributed under the Creative Commons Attribution License, which permits unrestricted use, distribution, and reproduction in any medium, provided the original work is properly cited.

\section{Introduction}

The stability problem of functional equations originated from a question of Ulam [1] concerning the stability of group homomorphisms. Hyers [2] gave a first affirmative answer to the question of Ulam for Banach spaces. Hyers' theorem was generalized by Aoki [3] for additive mapping and by Th. M. Rassias [4] for linear mappings by considering an unbounded Cauchy difference. The paper of Th. M. Rassias [4] has provided a lot of influence in the development of what we now call Hyers-Ulam-Rassias stability of functional equations. Th. M. Rassias [5] during the 27th International Symposium on Functional Equations asked the question whether such a theorem can also be proved for $p \geq 1$. Gajda [6], following the same approach as in [4], gave an affirmative solution to this question for $p>1$. It was shown by Gajda [6] as well as by Rassias and Šemrl [7] that one cannot prove a Th.M. Rassias' type theorem when $p=1$. J. M. Rassias [8], following the spirit of the innovative approach of Th. M. Rassias [4] for the unbounded Cauchy difference, proved a similar stability theorem in which he replaced the factor $\|x\|^{p}+\|y\|^{p}$ by $\|x\|^{p} \cdot\|y\|^{q}$ for $p, q \in \mathbb{R}$ with $p+q \neq 1$.

The functional equation

$$
f(x+y)+f(x-y)=2 f(x)+2 f(y)
$$

is called a quadratic functional equation. In particular, every solution of the quadratic functional 
equation is said to be a quadratic function. A Hyers-Ulam-Rassias stability problem for the quadratic functional equation was proved by Skof [9] for mappings $f: X \rightarrow Y$, where $X$ is a normed space and $Y$ is a Banach space. Cholewa [10] noticed that the theorem of Skof is still true if the relevant domain $X$ is replaced by an Abelian group. In [11], Czerwik proved the Hyers-Ulam-Rassias stability of the quadratic functional equation. Several functional equations have been investigated in [12-17].

Throughout this paper, assume that $k$ is a fixed positive integer.

In this paper, we solve the functional equation

$$
f(k x+y)+f(k x-y)=2 k^{2} f(x)+2 f(y)
$$

and prove the Hyers-Ulam-Rassias stability of the functional equation (1.2) in Banach spaces.

\section{Hyers-Ulam-Rassias stability of the quadratic functional equation}

Proposition 2.1. Let $X$ and $Y$ be vector spaces. A mapping $f: X \rightarrow Y$ satisfies

$$
f(k x+y)+f(k x-y)=2 k^{2} f(x)+2 f(y)
$$

for all $x, y \in X$ if and only if the mapping $f: X \rightarrow Y$ satisfies

$$
f(x+y)+f(x-y)=2 f(x)+2 f(y)
$$

for all $x, y \in X$.

Proof. Assume that $f: X \rightarrow Y$ satisfies (2.1).

Letting $x=y=0$ in (2.1), we get $f(0)=0$.

Letting $y=0$ in (2.1), we get $f(k x)=k^{2} f(x)$ for all $x \in X$.

Letting $x=0$ in (2.1), we get $f(-y)=f(y)$ for all $y \in X$.

It follows from (2.1) that

$$
f(k x+y)+f(k x-y)=2 k^{2} f(x)+2 f(y)=2 f(k x)+2 f(y)
$$

for all $x, y \in X$. So the mapping $f: X \rightarrow Y$ satisfies

$$
f(x+y)+f(x-y)=2 f(x)+2 f(y)
$$

for all $x, y \in X$.

Assume that $f: X \rightarrow Y$ satisfies $f(x+y)+f(x-y)=2 f(x)+2 f(y)$ for all $x, y \in X$.

We prove (2.1) for $k=j$ by induction on $j$.

For the case $j=1,(2.1)$ holds by the assumption.

For the case $j=2$, since

$$
\begin{aligned}
f(2 x+y)+f(2 x-y) & =f(x+y+x)+f(x-y+x) \\
& =2 f(x+y)+2 f(x)-f(y)+2 f(x-y)+2 f(x)-f(-y) \\
& =2 f(x+y)+2 f(x-y)+4 f(x)-2 f(y) \\
& =4 f(x)+4 f(y)+4 f(x)-2 f(y) \\
& =8 f(x)+2 f(y)
\end{aligned}
$$

for all $x, y \in X$, then (2.1) holds. 
Jung Rye Lee et al.

Assume that (2.1) holds for $j=n-2$ and $j=n-1(2<n \leq k)$. By the assumption,

$$
\begin{aligned}
f(n x+y)+f(n x-y)= & f((n-1) x+y+x)+f((n-1) x-y+x) \\
= & 2 f((n-1) x+y)+2 f(x)-f((n-2) x+y) \\
& +2 f((n-1) x-y)+2 f(x)-f((n-2) x-y) \\
= & 4(n-1)^{2} f(x)+4 f(y)+4 f(x)-2(n-2)^{2} f(x)-2 f(y) \\
= & 2 n^{2} f(x)+2 f(y)
\end{aligned}
$$

for all $x, y \in X,(2.1)$ holds for $j=n$. Hence the mapping $f: X \rightarrow Y$ satisfies (2.1) for $j=k$.

From now on, assume that $X$ is a normed vector space with norm $\|\cdot\|$ and that $Y$ is a Banach space with norm $\|\cdot\|$.

For a given mapping $f: X \rightarrow Y$, we define

$$
D f(x, y):=f(k x+y)+f(k x-y)-2 k^{2} f(x)-2 f(y)
$$

for all $x, y \in X$.

Now we prove the Hyers-Ulam-Rassias stability of the quadratic functional equation $D f(x, y)=0$.

Theorem 2.2. Let $f: X \rightarrow Y$ be a mapping with $f(0)=0$ for which there exists a function $\varphi: X^{2} \rightarrow$ $[0, \infty)$ such that

$$
\begin{gathered}
\tilde{\varphi}(x, y):=\sum_{j=0}^{\infty} \frac{1}{k^{2 j}} \varphi\left(k^{j} x, k^{j} y\right)<\infty, \\
\|D f(x, y)\| \leq \varphi(x, y)
\end{gathered}
$$

for all $x, y \in X$. Then there exists a unique quadratic mapping $Q: X \rightarrow Y$ such that

$$
\|f(x)-Q(x)\| \leq \frac{1}{2 k^{2}} \tilde{\varphi}(x, 0)
$$

for all $x \in X$.

Proof. Letting $y=0$ in (2.9), we get

$$
\left\|2 f(k x)-2 k^{2} f(x)\right\| \leq \varphi(x, 0)
$$

for all $x \in X$. So

$$
\left\|f(x)-\frac{1}{k^{2}} f(k x)\right\| \leq \frac{1}{2 k^{2}} \varphi(x, 0)
$$

for all $x \in X$. Hence

$$
\left\|\frac{1}{k^{2 l}} f\left(k^{l} x\right)-\frac{1}{k^{2 m}} f\left(k^{m} x\right)\right\| \leq \sum_{j=l}^{m-1} \frac{1}{2 k^{2 j+2}} \varphi\left(k^{j} x, 0\right)
$$


for all nonnegative integers $m$ and $l$ with $m>l$ and all $x \in X$. It follows from (2.13) that the sequence $\left\{\left(1 / k^{2 n}\right) f\left(k^{n} x\right)\right\}$ is Cauchy for all $x \in X$. Since $Y$ is complete, the sequence $\left\{\left(1 / k^{2 n}\right) f\left(k^{n} x\right)\right\}$ converges. So one can define the mapping $Q: X \rightarrow Y$ by

$$
Q(x):=\lim _{n \rightarrow \infty} \frac{1}{k^{2 n}} f\left(k^{n} x\right)
$$

for all $x \in X$.

By (2.8),

$$
\|D Q(x, y)\|=\lim _{n \rightarrow \infty} \frac{1}{k^{2 n}}\left\|D f\left(k^{n} x, k^{n} y\right)\right\| \leq \lim _{n \rightarrow \infty} \frac{1}{k^{2 n}} \varphi\left(k^{n} x, k^{n} y\right)=0
$$

for all $x, y \in X$. So $D Q(x, y)=0$. By Proposition 2.1, the mapping $Q: X \rightarrow Y$ is quadratic. Moreover, letting $l=0$ and passing the limit $m \rightarrow \infty$ in (2.13), we get (2.10).

Now, let $T: X \rightarrow Y$ be another quadratic mapping satisfying (2.1) and (2.10). Then we have

$$
\begin{aligned}
\|Q(x)-T(x)\| & =\frac{1}{k^{2 n}}\left\|Q\left(k^{n} x\right)-T\left(k^{n} x\right)\right\| \\
& \leq \frac{1}{k^{2 n}}\left(\left\|Q\left(k^{n} x\right)-f\left(k^{n} x\right)\right\|+\left\|T\left(k^{n} x\right)-f\left(k^{n} x\right)\right\|\right) \\
& \leq \frac{1}{k^{2 n+2}} \tilde{\varphi}\left(k^{n} x, 0\right),
\end{aligned}
$$

which tends to zero as $n \rightarrow \infty$ for all $x \in X$. So we can conclude that $Q(x)=T(x)$ for all $x \in X$. This proves the uniqueness of $Q$. So there exists a unique quadratic mapping $Q: X \rightarrow Y$ satisfying (2.10).

Corollary 2.3. Let $p<2$ and $\theta$ be positive real numbers, and let $f: X \rightarrow Y$ be a mapping such that

$$
\|D f(x, y)\| \leq \theta\left(\|x\|^{p}+\|y\|^{p}\right)
$$

for all $x, y \in X$. Then there exists a unique quadratic mapping $Q: X \rightarrow Y$ such that

$$
\|f(x)-Q(x)\| \leq \frac{\theta}{8-2^{p+1}}\|x\|^{p}
$$

for all $x \in X$.

Proof. The proof follows from Theorem 2.2 by taking

$$
\varphi(x, y):=\theta\left(\|x\|^{p}+\|y\|^{p}\right)
$$

for all $x, y \in A$.

Theorem 2.4. Let $f: X \rightarrow Y$ be a mapping with $f(0)=0$ for which there exists a function $\varphi: X^{2} \rightarrow$ $[0, \infty)$ satisfying $(2.9)$ such that

$$
\tilde{\varphi}(x, y):=\sum_{j=0}^{\infty} k^{2 j} \varphi\left(\frac{x}{k^{j}}, \frac{y}{k^{j}}\right)<\infty
$$

for all $x, y \in X$. Then there exists a unique quadratic mapping $Q: X \rightarrow Y$ such that

$$
\|f(x)-Q(x)\| \leq \frac{1}{2} \tilde{\varphi}\left(\frac{x}{k^{\prime}}, 0\right)
$$

for all $x \in X$. 
Proof. It follows from (2.11) that

$$
\left\|f(x)-k^{2} f\left(\frac{x}{k}\right)\right\| \leq \frac{1}{2} \varphi\left(\frac{x}{k}, 0\right)
$$

for all $x \in X$. Hence

$$
\left\|k^{2 l} f\left(\frac{x}{k^{l}}\right)-k^{2 m} f\left(\frac{x}{k^{m}}\right)\right\| \leq \sum_{j=l}^{m-1} \frac{k^{2 j}}{2} \varphi\left(\frac{x}{k^{j+1}}, 0\right)
$$

for all nonnegative integers $m$ and $l$ with $m>l$ and all $x \in X$. It follows from (2.23) that the sequence $\left\{k^{2 n} f\left(x / k^{n}\right)\right\}$ is Cauchy for all $x \in X$. Since $Y$ is complete, the sequence $\left\{k^{2 n} f\left(x / k^{n}\right)\right\}$ converges. So one can define the mapping $Q: X \rightarrow Y$ by

$$
Q(x):=\lim _{n \rightarrow \infty} k^{2 n} f\left(\frac{x}{k^{n}}\right)
$$

for all $x \in X$.

By (2.20),

$$
\|D Q(x, y)\|=\lim _{n \rightarrow \infty} k^{2 n}\left\|D f\left(\frac{x}{k^{n}}, \frac{y}{k^{n}}\right)\right\| \leq \lim _{n \rightarrow \infty} k^{2 n} \varphi\left(\frac{x}{k^{n}}, \frac{y}{k^{n}}\right)=0
$$

for all $x, y \in X$. So $D Q(x, y)=0$. By Proposition 2.1, the mapping $Q: X \rightarrow Y$ is quadratic. Moreover, letting $l=0$ and passing the limit $m \rightarrow \infty$ in (2.23), we get (2.21).

The rest of the proof is similar to the proof of Theorem 2.2.

Corollary 2.5. Let $p>2$ and $\theta$ be positive real numbers, and let $f: X \rightarrow Y$ be a mapping satisfying (2.17). Then there exists a unique quadratic mapping $Q: X \rightarrow Y$ such that

$$
\|f(x)-Q(x)\| \leq \frac{\theta}{2^{p+1}-8}\|x\|^{p}
$$

for all $x \in X$.

Proof. The proof follows from Theorem 2.4 by taking

$$
\varphi(x, y):=\theta\left(\|x\|^{p}+\|y\|^{p}\right)
$$

for all $x, y \in A$.

From now on, assume that $k=2$.

Theorem 2.6. Let $f: X \rightarrow Y$ be a mapping with $f(0)=0$ for which there exists a function $\varphi: X^{2} \rightarrow$ $[0, \infty)$ satisfying $(2.9)$ such that

$$
\tilde{\varphi}(x, y):=\sum_{j=0}^{\infty} \frac{1}{9^{j}} \varphi\left(3^{j} x, 3^{j} y\right)<\infty
$$

for all $x, y \in X$. Then there exists a unique quadratic mapping $Q: X \rightarrow Y$ such that

$$
\|f(x)-Q(x)\| \leq \frac{1}{9} \tilde{\varphi}(x, x)
$$

for all $x \in X$. 
Proof. Letting $y=x$ in (2.9), we get

$$
\|f(3 x)-9 f(x)\| \leq \varphi(x, x)
$$

for all $x \in X$. So

$$
\left\|f(x)-\frac{1}{9} f(3 x)\right\| \leq \frac{1}{9} \varphi(x, x)
$$

for all $x \in X$. Hence

$$
\left\|\frac{1}{9^{l}} f\left(3^{l} x\right)-\frac{1}{9^{m}} f\left(3^{m} x\right)\right\| \leq \sum_{j=l}^{m-1} \frac{1}{9^{j+1}} \varphi\left(3^{j} x, 3^{j} x\right)
$$

for all nonnegative integers $m$ and $l$ with $m>l$ and all $x \in X$. It follows from (2.32) that the sequence $\left\{\left(1 / 9^{n}\right) f\left(3^{n} x\right)\right\}$ is Cauchy for all $x \in X$. Since $Y$ is complete, the sequence $\left\{\left(1 / 9^{n}\right) f\left(3^{n} x\right)\right\}$ converges. So one can define the mapping $Q: X \rightarrow Y$ by

$$
Q(x):=\lim _{n \rightarrow \infty} \frac{1}{9^{n}} f\left(3^{n} x\right)
$$

for all $x \in X$.

By (2.28),

$$
\|D Q(x, y)\|=\lim _{n \rightarrow \infty} \frac{1}{9^{n}}\left\|D f\left(3^{n} x, 3^{n} y\right)\right\| \leq \lim _{n \rightarrow \infty} \frac{1}{9^{n}} \varphi\left(3^{n} x, 3^{n} y\right)=0
$$

for all $x, y \in X$. So $D Q(x, y)=0$. By Proposition 2.1, the mapping $Q: X \rightarrow Y$ is quadratic. Moreover, letting $l=0$ and passing the limit $m \rightarrow \infty$ in (2.32), we get (2.29).

The rest of the proof is similar to the proof of Theorem 2.2.

Corollary 2.7. Let $p<1$ and $\theta$ be positive real numbers, and let $f: X \rightarrow Y$ be a mapping such that

$$
\|D f(x, y)\| \leq \theta \cdot\|x\|^{p} \cdot\|y\|^{p}
$$

for all $x, y \in X$. Then there exists a unique quadratic mapping $Q: X \rightarrow Y$ such that

$$
\|f(x)-Q(x)\| \leq \frac{\theta}{9-9^{p}}\|x\|^{2 p}
$$

for all $x \in X$.

Proof. The proof follows from Theorem 2.6 by taking

$$
\varphi(x, y):=\theta \cdot\|x\|^{p} \cdot\|y\|^{p}
$$

for all $x, y \in A$. 
Theorem 2.8. Let $f: X \rightarrow Y$ be a mapping with $f(0)=0$ for which there exists a function $\varphi: X^{2} \rightarrow$ $[0, \infty)$ satisfying $(2.9)$ such that

$$
\tilde{\varphi}(x, y):=\sum_{j=0}^{\infty} 9^{j} \varphi\left(\frac{x}{3^{j}}, \frac{y}{3^{j}}\right)<\infty
$$

for all $x, y \in X$. Then there exists a unique quadratic mapping $Q: X \rightarrow Y$ such that

$$
\|f(x)-Q(x)\| \leq \tilde{\varphi}\left(\frac{x}{3}, \frac{x}{3}\right)
$$

for all $x \in X$.

Proof. It follows from (2.30) that

$$
\left\|f(x)-9 f\left(\frac{x}{3}\right)\right\| \leq \varphi\left(\frac{x}{3}, \frac{x}{3}\right)
$$

for all $x \in X$. Hence

$$
\left\|9^{l} f\left(\frac{x}{3^{l}}\right)-9^{m} f\left(\frac{x}{3^{m}}\right)\right\| \leq \sum_{j=l}^{m-1} 9^{j} \varphi\left(\frac{x}{3^{j+1}}, \frac{x}{3^{j+1}}\right)
$$

for all nonnegative integers $m$ and $l$ with $m>l$ and all $x \in X$. It follows from (2.41) that the sequence $\left\{9^{n} f\left(x / 3^{n}\right)\right\}$ is Cauchy for all $x \in X$. Since $Y$ is complete, the sequence $\left\{9^{n} f\left(x / 3^{n}\right)\right\}$ converges. So one can define the mapping $Q: X \rightarrow Y$ by

$$
Q(x):=\lim _{n \rightarrow \infty} 9^{n} f\left(\frac{x}{3^{n}}\right)
$$

for all $x \in X$.

By (2.38),

$$
\|D Q(x, y)\|=\lim _{n \rightarrow \infty} \frac{1}{9^{n}}\left\|D f\left(3^{n} x, 3^{n} y\right)\right\| \leq \lim _{n \rightarrow \infty} \frac{1}{9^{n}} \varphi\left(3^{n} x, 3^{n} y\right)=0
$$

for all $x, y \in X$. So $D Q(x, y)=0$. By Proposition 2.1, the mapping $Q: X \rightarrow Y$ is quadratic. Moreover, letting $l=0$ and passing the limit $m \rightarrow \infty$ in (2.41), we get (2.39).

The rest of the proof is similar to the proof of Theorem 2.2.

Corollary 2.9. Let $p>1$ and $\theta$ be positive real numbers, and let $f: X \rightarrow Y$ be a mapping satisfying (2.35). Then there exists a unique quadratic mapping $Q: X \rightarrow Y$ such that

$$
\|f(x)-Q(x)\| \leq \frac{\theta}{9^{p}-9}\|x\|^{2 p}
$$

for all $x \in X$.

Proof. The proof follows from Theorem 2.8 by taking

$$
\varphi(x, y):=\theta \cdot\|x\|^{p} \cdot\|y\|^{p}
$$

for all $x, y \in A$. 


\section{Acknowledgments}

Jung Rye Lee was supported by Daejin University grants in 2007. The authors would like to thank the referees for a number of valuable suggestions regarding a previous version of this paper.

\section{References}

[1] S. M. Ulam, Problems in Modern Mathematics, John Wiley \& Sons, New York, NY, USA, 1960.

[2] D. H. Hyers, "On the stability of the linear functional equation," Proceedings of the National Academy of Sciences of the United States of America, vol. 27, no. 4, pp. 222-224, 1941.

[3] T. Aoki, "On the stability of the linear transformation in Banach spaces," Journal of the Mathematical Society of Japan, vol. 2, pp. 64-66, 1950.

[4] Th. M. Rassias, "On the stability of the linear mapping in Banach spaces," Proceedings of the American Mathematical Society , vol. 72, no. 2, pp. 297-300, 1978.

[5] Th. M. Rassias, "Problem 16; 2, Report of the 27th International Symposium on Functional Equations," Aequationes Mathematicae, vol. 39, no. 2-3, pp. 292-293, 309, 1990.

[6] Z. Gajda, "On stability of additive mappings," International Journal of Mathematics and Mathematical Sciences , vol. 14, no. 3, pp. 431-434, 1991.

[7] Th. M. Rassias and P. Šemrl, "On the Hyers-Ulam stability of linear mappings," Journal of Mathematical Analysis and Applications, vol. 173, no. 2, pp. 325-338, 1993.

[8] J. M. Rassias, "On approximation of approximately linear mappings by linear mappings," Bulletin des Sciences Mathématiques, vol. 108, no. 4, pp. 445-446, 1984.

[9] F. Skof, "Local properties and approximation of operators," Rendiconti del Seminario Matematico e Fisico di Milano, vol. 53, pp. 113-129, 1983.

[10] P. W. Cholewa, "Remarks on the stability of functional equations," Aequationes Mathematicae , vol. 27, no. 1, pp. 76-86, 1984.

[11] St. Czerwik, "On the stability of the quadratic mapping in normed spaces," Abhandlungen aus dem Mathematischen Seminar der Universität Hamburg, vol. 62, pp. 59-64, 1992.

[12] K.-W. Jun and Y.-H. Lee, "On the Hyers-Ulam-Rassias stability of a pexiderized quadratic inequality," Mathematical Inequalities \& Applications, vol. 4, no. 1, pp. 93-118, 2001.

[13] S.-M. Jung, "On the Hyers-Ulam stability of the functional equations that have the quadratic property," Journal of Mathematical Analysis and Applications , vol. 222, no. 1, pp. 126-137, 1998.

[14] J. M. Rassias, "Solution of a quadratic stability Hyers-Ulam type problem," Ricerche di Matematica , vol. 50, no. 1, pp. 9-17, 2001.

[15] J. M. Rassias, "On approximation of approximately quadratic mappings by quadratic mappings," Annales Mathematicae Silesianae , no. 15, pp. 67-78, 2001.

[16] M. Mirzavaziri and M. S. Moslehian, "A fixed point approach to stability of a quadratic equation," Bulletin of the Brazilian Mathematical Society , vol. 37, no. 3, pp. 361-376, 2006.

[17] Th. M. Rassias, "On the stability of the quadratic functional equation and its applications," Studia Universitatis Babeş-Bolyai , vol. 43, no. 3, pp. 89-124, 1998. 